International Journal of Pure and Applied Mathematics

Volume 87 No. 2 2013, 293-299

ISSN: 1311-8080 (printed version); ISSN: 1314-3395 (on-line version)

url: http://www.ijpam.eu

doi: http://dx.doi.org/10.12732/ijpam.v87i2.10

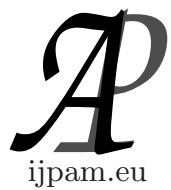

\title{
A NEW TYPE OF QUASI-OPEN AND QUASI-CLOSED FUNCTIONS
}

\author{
N. Rajesh ${ }^{1}$, G. Shanmugam ${ }^{2}$ \\ ${ }^{1}$ Department of Mathematics \\ Rajah Serfoji Govt. College \\ Thanjavur, 613005, Tamilnadu, INDIA \\ ${ }^{2}$ Department of Mathematics \\ Jeppiaar Engineering College \\ Chennai, 600119, Tamilnadu, INDIA
}

\begin{abstract}
The purpose of this paper is to give a new type of open function called, quasi $s g \alpha$-open function. Also we obtain its characterizations and its basic properties.
\end{abstract}

AMS Subject Classification: 54C10, 54C08, 54C05

Key Words: topological spaces, $s g \alpha$-open sets, quasi $\operatorname{sg} \alpha$-open functions

\section{Introduction and Preliminaries}

Functions and of course open functions stand among the most important notions in the whole of mathematical science. Many different forms of open functions have been introduced over the years. Various interesting problems arise when one considers openness. Its importance is significant in various areas of mathematics and related sciences. Recently, as a generalization of closed sets, the notion of $s g \alpha$-closed sets were introduced and studied by Rajesh and Krsteska [6]. In this paper, we will continue the study of related functions by involving $s g \alpha$-open sets. We introduce and characterize the concept of quasi$s g \alpha$-open functions. Throughout this paper, spaces means topological spaces

Received: April 11, 2013

(C) 2013 Academic Publications, Ltd.

$\S$ Correspondence author url: www.acadpubl.eu 
on which no separation axioms are assumed unless otherwise mentioned and $f:(X, \tau) \rightarrow(Y, \sigma)$ (or simply $f: X \rightarrow Y$ ) denotes a function $f$ of a space $(X, \tau)$ into a space $(Y, \sigma)$. Let $A$ be a subset of a space $X$. The closure and the interior of $A$ are denoted by $\mathrm{Cl}(A)$ and $\operatorname{Int}(A)$, respectively.

Definition 1. A subset $A$ of a space $(X, \tau)$ is called semi-open [2] (resp. $\alpha$-open [3]) if $A \subset \mathrm{Cl}(\operatorname{Int}(A))$ (resp. $A \subset \operatorname{Int}(\mathrm{Cl}(\operatorname{Int}(A)))$ ). The complement of a semi-open (resp. $\alpha$-open) set is called semi-closed (resp. $\alpha$-closed).

The semi-closure [1] of a subset $A$ of $X$, denoted by $s \mathrm{Cl}(A)$, is defined to be the intersection of all semi-closed sets containing $A$ in $X$. The $\alpha$-closure of a subset is similarly defined.

Definition 2. A subset $A$ of a space $X$ is called semi-generalized closed (briefly sg $\alpha$-closed) [6] if $\alpha \mathrm{Cl}(A) \subset U$ whenever $A \subset U$ and $U$ is semi-open in $X$. The complement of $s g \alpha$-closed set is called $s g \alpha$-open.

The union (resp. intersection) of all $s g \alpha$-open (resp. $s g \alpha$-closed) sets, each contained in (resp. containing) a set $A$ in a space $X$ is called the $s g \alpha$-interior (resp. $s g \alpha$-closure) of $A$ and is denoted by $s g \alpha$ - $\operatorname{Int}(A)$ (resp. $s g \alpha-\mathrm{Cl}(A))[6]$.

Definition 3. A function $f:(X, \tau) \rightarrow(Y, \sigma)$ is called:

(i) $s g \alpha$-irresolute [4] (sg $\alpha$-continuous [7]) if $f^{-1}(V)$ is $s g \alpha$-closed in $X$ for every $s g \alpha$-closed (resp. closed) subset $V$ of $Y$;

(ii) $s g \alpha$-open [4] (resp. sg $\alpha$-closed [4]) if $f(V)$ is $s g \alpha$-open (resp. $s g \alpha$-closed) in $Y$ for every open (resp. closed) subset of $X$.

\section{Quasi $s g \alpha$-Open Functions}

Definition 4. A function $f: X \rightarrow Y$ is said to be quasi $s g \alpha$-open if the image of every $s g \alpha$-open set in $X$ is open in $Y$.

It is evident that, the concepts quasi $s g \alpha$-openness and $s g \alpha$-continuity coincide if the function is a bijection.

Theorem 5. A function $f: X \rightarrow Y$ is quasi-sgo-open if and only if for every subset $U$ of $X, f(s g \alpha-\operatorname{Int}(U)) \subset \operatorname{Int}(f(U))$.

Proof. Let $f$ be quasi sga-open function. Now, we have $\operatorname{Int}(U) \subset U$ and $s g \alpha$-Int $(U)$ is a $s g \alpha$-open set. Hence, we obtain that $f(s g \alpha-\operatorname{Int}(U)) \subset f(U)$. As $f(s g \alpha-\operatorname{Int}(U))$ is open, then $f(s g \alpha-\operatorname{Int}(U)) \subset \operatorname{Int}(f(U))$. Conversely, assume 
that $U$ be a $s g \alpha$-open set in $X$. Then, $f(U)=f(s g \alpha-\operatorname{Int}(U)) \subset \operatorname{Int}(f(U))$ but usually $\operatorname{Int}(f(U)) \subset f(U)$. Consequently, $f(U)=\operatorname{Int}(f(U))$ and hence $f$ is quasi $s g \alpha$-open.

Lemma 6. A function $f: X \rightarrow Y$ is quasi sg $\alpha$-open, then $\operatorname{sg} \alpha-\operatorname{Int}\left(f^{-1}(G)\right)$ $\subset f^{-1}(\operatorname{Int}(G))$ for every subset $G$ of $Y$.

Proof. Let $G$ be any arbitrary subset of $Y$. Then, $s g \alpha-\operatorname{Int}\left(f^{-1}(G)\right)$ is a $s g \alpha$-open set in $X$ and $f$ is quasi $s g \alpha$-open, then $f\left(\operatorname{sg} \alpha\right.$ - $\left.\operatorname{Int}\left(f^{-1}(G)\right)\right) \subset$ $\operatorname{Int}\left(f\left(f^{-1}(G)\right)\right) \subset \operatorname{Int}(G)$. Thus, $s g \alpha-\operatorname{Int}\left(f^{-1}(G)\right) \subset f^{-1}(\operatorname{Int}(G))$.

Recall that a subset $S$ is called a $s g \alpha$-neighbourhood [7] of a point $x$ of $X$ if there exists a $s g \alpha$-open set $U$ such that $x \in U \subset S$.

Theorem 7. For a function $f: X \rightarrow Y$, the following are equivalent:

(i) $f$ is quasi sgo-open;

(ii) For each subset $U$ of $X, f(s g \alpha-\operatorname{Int}(U)) \subset \operatorname{Int}(f(U))$;

(iii) For each $x \in X$ and each sgo-neighbourhood $U$ of $x$ in $X$, there exists a neighbourhood $V$ of $f(x)$ in $Y$ such that $V \subset f(U)$.

Proof. (i) $\Rightarrow$ (ii): It follows from Theorem 5. (ii) $\Rightarrow$ (iii): Let $x \in X$ and $U$ be an arbitrary $s g \alpha$-neighbourhood of $x$ in $X$. Then there exists a $s g \alpha$-open set $V$ in $X$ such that $x \in V \subset U$. Then by (ii), we have $f(V)=f(s g \alpha-\operatorname{Int}(V)) \subset$ $\operatorname{Int}(f(U))$ and hence $f(V)=\operatorname{Int}(f(V))$. Therefore, it follows that $f(V)$ is open in $Y$ such that $f(x) \in f(V) \subset f(U)$. (iii) $\Rightarrow($ i): Let $U$ be an arbitrary $s g \alpha$-open set in $X$. Then for each $y \in f(U)$, by (iii) there exists a neighbourhood $V_{y}$ of $y$ in $Y$ such that $V_{y} \subset f(U)$. As $V_{y}$ is a neighbourhood of $y$, there exists an open set $W_{y}$ in $Y$ such that $y \in W_{y} \subset V_{y}$. Thus, $f(U)=\bigcup\left\{W_{y}: y \in f(U)\right\}$ which is an open set in $Y$. This implies that $f$ is quasi $s g \alpha$-open function.

Theorem 8. A function $f: X \rightarrow Y$ is quasi sgo-open if and only if for any subset $B$ of $Y$ and for any sg $\alpha$-closed set $F$ of $X$ containing $f^{-1}(B)$, there exists a closed set $G$ of $Y$ containing $B$ such that $f^{-1}(G) \subset F$.

Proof. Suppose $f$ is quasi $s g \alpha$-open. Let $B \subset Y$ and $F$ be a $s g \alpha$-closed set of $X$ containing $f^{-1}(B)$. Now, put $G=Y-f(X-F)$. It is clear that $f^{-1}(B) \subset F$ implies $B \subset G$. Since $f$ is quasi $s g \alpha$-open, we obtain $G$ as a closed set of $Y$. Then, we have $f^{-1}(G) \subset F$. Conversely, let $U$ be a $s g \alpha$-open set of $X$ and put $B=Y-f(U)$. Then $X-U$ is a $s g \alpha$-closed set in $X$ containing $f^{-1}(B)$. By hypothesis, there exists a closed set $F$ of $Y$ such that $B \subset F$ and 
$f^{-1}(F) \subset X-U$. Hence, we obtain $f(U) \subset Y-F$. On the other hand, it follows that $B \subset F, Y-F \subset Y-B=f(U)$. Thus, we obtain $f(U)=Y-F$ which is open and hence $f$ is a quasi $s g \alpha$-open function.

Theorem 9. A function $f: X \rightarrow Y$ is quasi sgo-open if and only if $f(\mathrm{Cl}(B)) \subset \operatorname{sg} \alpha-\mathrm{Cl}(f(B))$ for every subset $B$ of $Y$.

Proof. Suppose that $f$ is quasi $s g \alpha$-open. For any subset $B$ of $Y, f^{-1}(B) \subset$ $s g \alpha-\mathrm{Cl}\left(f^{-1}(B)\right)$. Therefore by Theorem 8, there exists a closed set $F$ in $Y$ such that $B \subset F$ and $f^{-1}(F) \subset s g \alpha-C l\left(f^{-1}(B)\right)$. Therefore, we obtain $f^{-1}(\mathrm{Cl}(B))$ $\subset f^{-1}(F) \subset s g \alpha-\mathrm{Cl}\left(f^{-1}(B)\right)$. Conversely, let $B \subset Y$ and $F$ be a $s g \alpha$-closed set of $X$ containing $f^{-1}(B)$. Put $W=\mathrm{Cl}_{Y}(B)$, then we have $B \subset W$ and $W$ is closed and $f^{-1}(W) \subset s g \alpha-\mathrm{Cl}\left(f^{-1}(B)\right) \subset F$. Then by Theorem $8, f$ is quasi sg $\alpha$-open.

Lemma 10. Let $f: X \rightarrow Y$ and $g: Y \rightarrow Z$ be two functions and $g \circ f:$

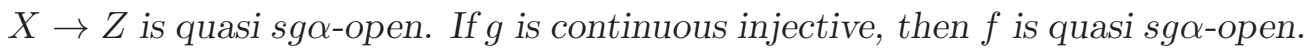

Proof. Let $U$ be a $s g \alpha$-open set in $X$, then $(g \circ f)(U)$ is open in $Z$ since $g \circ f$ is quasi $s g \alpha$-open. Again $g$ is an injective continuous function, $f(U)=$ $g^{-1}(g \circ f(U))$ is open in $Y$. This shows that $f$ is quasi $s g \alpha$-open.

\section{Quasi $s g \alpha$-Closed Functions}

Definition 11. A function $f: X \rightarrow Y$ is said to be quasi $s g \alpha$-closed if the image of each $s g \alpha$-closed set in $X$ is closed in $Y$.

Clearly, every quasi $s g \alpha$-closed function is closed as well as $s g \alpha$-closed.

Remark 12. Every $s g \alpha$-closed (resp. closed) function need not be quasi $s g \alpha$-closed as shown by the following example.

Example 13. Let $X=Y=\{a, b, c\}, \tau=\{\emptyset,\{a, b\}, X\}$ and $\sigma=\{\emptyset,\{a\}$, $\{b, c\}, Y\}$. Define a function $f:(X, \tau) \rightarrow(Y, \sigma)$ by $f(a)=b, f(b)=c$ and $f(c)$ $=a$. Then clearly $f$ is $s g \alpha$-closed as well as closed but not quasi $s g \alpha$-closed.

Lemma 14. A function $f: X \rightarrow Y$ is quasi sgo-closed if and only if $f^{-1}(\operatorname{Int}(B)) \subset \operatorname{sg} \alpha-\operatorname{Int}\left(f^{-1}(B)\right)$ for every subset $B$ of $Y$.

Proof. This proof is similar to the proof of Lemma 6 . 
Theorem 15. A function $f: X \rightarrow Y$ is quasi sgo-closed if and only if for any subset $B$ of $Y$ and for any sgo-open set $G$ of $X$ containing $f^{-1}(B)$, there exists an open set $U$ of $Y$ containing $B$ such that $f^{-1}(U) \subset G$.

Proof. This proof is similar to that of Theorem 8 .

Remark 16. Let $f: X \rightarrow Y$ be a quasi $s g \alpha$-closed function. If $Y$ is a $T_{s g \alpha}$-space, then quasi $s g \alpha$-closedness coincide with $s g \alpha^{*}$-closedness.

Theorem 17. If $f: X \rightarrow Y$ and $g: Y \rightarrow Z$ are two quasi sgo-closed function, then $g \circ f: X \rightarrow Z$ is a quasi sgo-closed function.

Proof. Obvious.

Definition 18. A function $f: X \rightarrow Y$ is called $s g \alpha^{*}$-closed [4] if the image of every $s g \alpha$-closed subset of $X$ is $s g \alpha$-closed in $Y$.

Theorem 19. Let $f: X \rightarrow Y$ and $g: Y \rightarrow Z$ be any two functions. Then

(i) If $f$ is $s g \alpha$-closed and $g$ is quasi $s g \alpha$-closed, then $g \circ f$ is closed;

(ii) If $f$ is quasi $s g \alpha$-closed and $g$ is $s g \alpha$-closed, then $g \circ f$ is sgo*-closed;

(iii) If $f$ is $s g \alpha^{*}$-closed and $g$ is quasi $s g \alpha$-closed, then $g \circ f$ is quasi sg $\alpha$-closed;

Proof. Obvious.

Theorem 20. Let $f: X \rightarrow Y$ and $g: Y \rightarrow Z$ be two functions such that $g \circ f: X \rightarrow Z$ is quasi sgo-closed.

(i) If $f$ is $s g \alpha$-irresolute surjective, then $g$ is closed.

(ii) If $g$ is $s g \alpha$-continuous injective, then $f$ is $s g \alpha^{*}$-closed.

Proof. (i) Suppose $F$ is an arbitrary $s g \alpha$-closed set in $Y$. As $f$ is $s g \alpha$ irresolute, $f^{-1}(F)$ is $s g \alpha$-closed in $X$. Since $g \circ f$ is quasi $s g \alpha$-closed and $f$ is surjective, $\left(g \circ f\left(f^{-1}(F)\right)\right)=g(F)$, which is closed in $Z$. This implies that $g$ is a closed function.

(ii) Suppose $F$ is any $s g \alpha$-closed set in $X$. Since $g \circ f$ is quasi $s g \alpha$-closed, $(g \circ f)(F)$ is closed in $Z$. Again $g$ is a $s g \alpha$-continuous injective function, $g^{-1}(g \circ$ $f(F))=f(F)$, which is $s g \alpha$-closed in $Y$. This shows that $f$ is $s g \alpha^{*}$-closed.

Theorem 21. Let $X$ and $Y$ be topological spaces. Then the function $g$ : $X \rightarrow Y$ is a quasi sgo-closed if and only $g(X)$ is closed in $Y$ and $g(V)-g(X-V)$ is open in $g(X)$ whenever $V$ is sga-open in $X$. 
Proof. Necessity: Suppose $g: X \rightarrow Y$ is a quasi $s g \alpha$-closed function. Since $X$ is $s g \alpha$-closed, $g(X)$ is closed in $Y$ and $g(V)-g(X-V)=g(X)-g(X-V)$ is open in $g(X)$ when $V$ is $s g \alpha$-open in $X$. Sufficiency: Suppose $g(X)$ is closed in $Y, g(V)-g(X-V)$ is open in $g(X)$ when $V$ is $s g \alpha$-open in $X$, and let $C$ be closed in $X$. Then $g(C)=g(X)-(g(X-C)-g(C))$ is closed in $g(X)$ and hence, closed in $Y$.

Corollary 22. Let $X$ and $Y$ be topological spaces. Then a surjective function $g: X \rightarrow Y$ is quasi sgo-closed if and only if $g(V)-g(X-V)$ is open in $Y$ whenever $U$ is sga-open in $X$.

Proof. Obvious.

Corollary 23. Let $X$ and $Y$ be topological spaces and let $g: X \rightarrow Y$ be

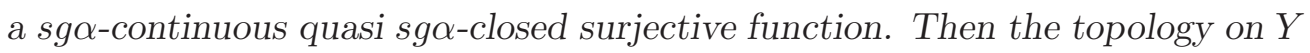
is $\{g(V)-g(X-V): V$ is sgo-open in $X\}$.

Proof. Let $W$ be open in $Y$. Then $g^{-1}(W)$ is $s g \alpha$-open in $X$, and $g\left(g^{-1}(W)\right)-$ $g\left(X-g^{-1}(W)\right)=W$. Hence, all open sets in $Y$ are of the form $g(V)-g(X-V)$, $V$ is $s g \alpha$-open in $X$. On the other hand, all sets of the form $g(V)-g(X-V)$, $V$ is $s g \alpha$-open in $X$, are open in $Y$ from Corollary 22.

Definition 24. A topological space $(X, \tau)$ is said to be $s g \alpha$-normal [5] if for any pair of disjoint $s g \alpha$-closed subsets $F_{1}$ and $F_{2}$ of $X$, there exist disjoint open sets $U$ and $V$ such that $F_{1} \subset U$ and $F_{2} \subset V$.

Theorem 25. Let $X$ and $Y$ be topological spaces with $X$ is sgo-normal

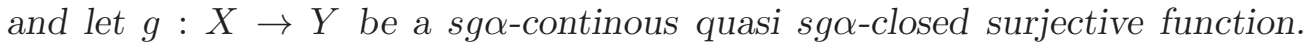
Then $Y$ is normal.

Proof. Let $K$ and $M$ be disjoint closed subsets of $Y$. Then $g^{-1}(K), g^{-1}(M)$ are disjoint $s g \alpha$-closed subsets of $X$. Since $X$ is $s g \alpha$-normal, there exist disjoint open sets $V$ and $W$ such that $g^{-1}(K) \subset V$ and $g^{-1}(M) \subset W$. Then $K \subset g(V)-$ $g(X-V)$ and $M \subset g(W)-g(X-W)$. Further by Corollary 22, $g(V)-g(X-V)$ and $g(W)-g(X-W)$ are open sets in $Y$ and clearly $(g(V)-g(X-V)) \bigcap$ $(g(W) g(X-W))=\emptyset$. This shows that $Y$ is normal.

\section{References}

[1] S.G. Crossley, S.K. Hildebrand, Semi-closure, Texas J. Sci., 22 (1971), 99-112. 
[2] N. Levine, Semi-open sets and semi-continuity in topological spaces, Amer. Math. Monthly., 70 (1963), 36-41.

[3] O. Njastad, On some classes of nearly open sets, Pacific J. Math., 15 (1965), 961-970.

[4] N. Rajesh, G. Shanmugam, On sgo-Homeomorphisms in topological spaces, Submitted.

[5] N. Rajesh, G. Shanmugam, On $s g \alpha$-regular space and $s g \alpha$-normal space, Submitted.

[6] N. Rajesh, B. Krsteska, Semigeneralized $\alpha$-closed sets, Antarctica J. Math., 6, No. 1 2009, 1-12.

[7] N. Rajesh, B. Krsteska, Semigeneralized $\alpha$-continuous functions, Submitted. 
